\title{
Root cause analysis of rough conical seat grinding problem in fuel pump cylinder head by Shainin methodology
}

\author{
Sanket Shankar Kadam ${ }^{1 *}$,Virupakshappa N. M. ${ }^{2}$ and U. Achutha Kini ${ }^{1}$ \\ ${ }^{1}$ Department of Mechanical and Manufacturing Engineering, Manipal Institute of Technology, \\ Manipal Academy of Higher Education, Manipal, Karnataka, India \\ ${ }^{2}$ Process planning- CPx components, Bosch Ltd., Bidadi, 562109, Karnataka, India
}

\begin{abstract}
Problem solving plays a major role in quality engineering and it is popularly adopted by manufacturing industry. This study is aimed to determine root cause for rejection of cylinder head due to rough conical grinding problem by Shainin method. This has two major parts, first is diagnostic part which involves finding out the root cause and second is a remedial action part which consist of actual application of findings to rule out the problem. Wear in tool holder assembly is identified as root cause for rough grinding problem. Converging to root cause and validating the same achieved by successful implementation of Shainin tools.
\end{abstract}

\section{Introduction}

Fuel injection pump is the important part of the common rail system (CRS). To generate the pressure demanded by CRS it is necessary to produce components of pump with highest quality .Problem solving comes under lean efforts taken by organization [1]. It plays a major role in both ways to improve quality and reduce the rejections and losses [2]. Shainin is convergent method of problem solving, involves continuously keep on eliminating the variables to converge to the most influencing variable [3]. Shainin methodology based on identifying contrast between good and bad pats, that's why it is simple and fast [4]. It makes use of specific tools to converge to the root cause quickly [5].

\section{Experimental approach: identification of root cause}

\subsection{Problem identification and understanding}

Cylinder head is one of the component for the CPx fuel injection pump, plunger reciprocates in the barrel which sucks fuel from low pressure (LP) port and delivers high pressure fuel from high pressure (HP) port. Conical grinding of HP port is done as illustrated in Fig. 1 for the depth of $40 \mu \mathrm{m}$. Tool can be lifted up by 0.20 to $0.40 \mathrm{~mm}$ with

* Corresponding author: sanket.kadam0200@gmail.com 
each one micron downward movement during grinding, this value can be manually controlled and known as tool lift off value. Scrap cost per annum and rework cost per annum is high for high pressure (HP) grinding operation than rejection from any other defect in cylinder head. Due to this problem statement selected was to find and eliminate root cause (Red X) for HP seat grinding rejections in cylinder head seat grinding process

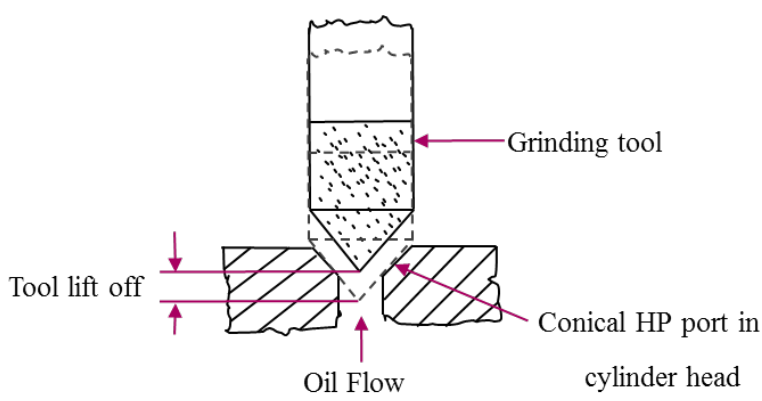

Fig. 1. Schematic representation of conical grinding operation

In HP seat grinding rejections, three kind of rejections are observed and those are rough lines on HP port, patches on HP port and cone offset. Amongst these, rejections due to rough lines as indicated in Fig. 2 are more than patches and cone offset. Rough lines on high pressure seat leads to leakage of diesel from high pressure area to low pressure area. Rough lines on HP port are indicated in Fig. 3.

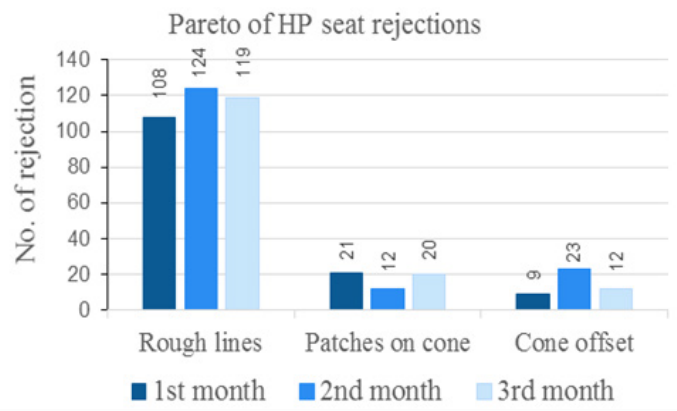

Fig. 2. Rejections Pareto

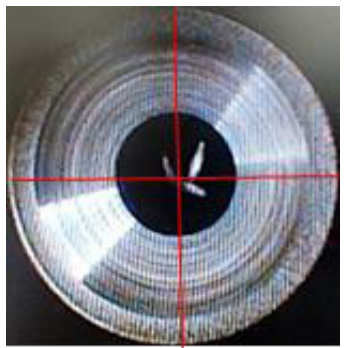

Fig. 3. Rough lines on HP port

\subsection{Clue generation}

Clue generation is done in two stages first one involves finding Green Y in Project definition tree and in second stage finding contrast between good and bad parts which further converges analysis. Project definition tree as shown in Fig.4, starts with project definition. Next step will be categorization of problem into one of the four categories Event, Feature, Property and Defect. In present study problem comes under defect category in which rough lines problem is dominant. Green Y (effect of problem on part) for problem is rough lines on HP seat. Clue generation in Shainin methodology is done by micro to macro approach. In second stage of clue generation, contrast is observed in families of variations such as, point to point, region to region, part to part etc. between best of best and worst of worst parts [6]. 


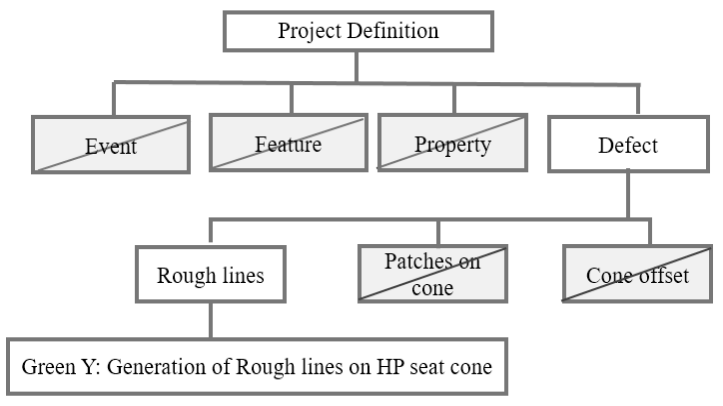

Fig. 4. Project definition tree

In this study contrast checked for region to region by dividing HP grinding face of cylinder head in four equal parts as shown in Fig. 3 and found that rough marks are there in all four sectors in rejected parts which indicates low contrast. There are three machines which are used for HP seat grinding operation, Machine to Machine rejection was checked and high variation was observed (Machine 1: 3\%, Machine 2: 2\%, Machine 3: 6\%).

\subsection{Root cause identification}

In Shainin methodology Green Y (effect of problem on part) is output and Red X (root cause) is input. For root cause identification tool used in Shainin methodology is solution tree. In solution tree shown in Fig.5, variables are eliminated one by one to identify most influencing variable amongst all. In first stage either measurement system variation $(\Delta \mathrm{M})$ or process variation $(\Delta \mathrm{P})$ has to be eliminated. Isoplot tool is utilized to check measurement variation, descrimination ratio more than 6.0 in Isoplot ensures elimination of measurement variation [7]. Here measurement variation is eliminated as descrimination ratio is 7.6 (more than 6.0) from Isoplot as shown in Fig.6.

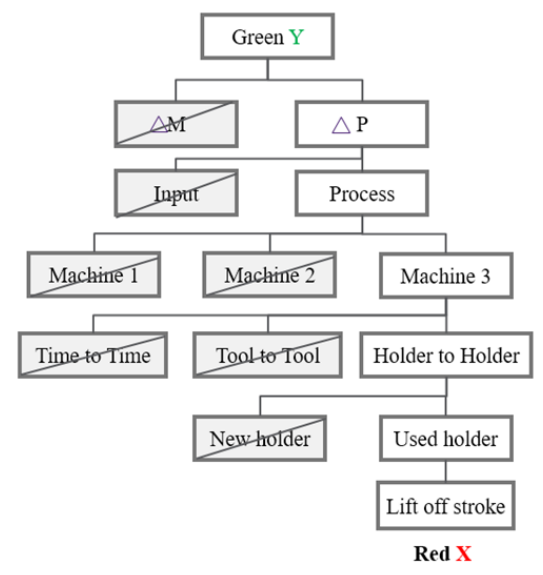

Fig. 5. Solution Tree

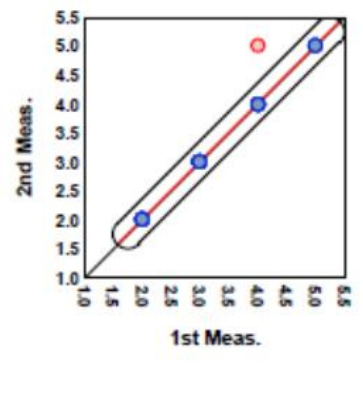

Fig. 6. Isoplot 


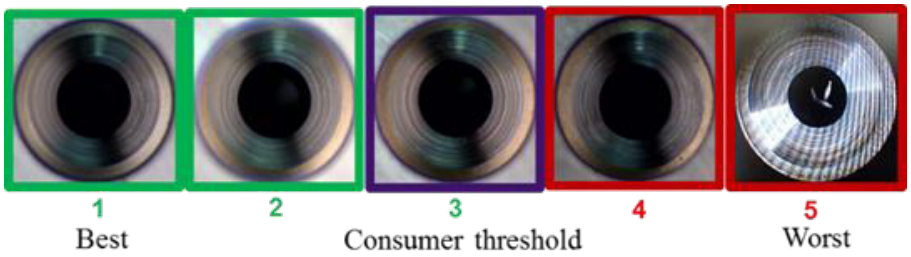

Fig. 7. Sensory scoring system

As defect is visual, for quantifying defect severity sensory scoring system is used as shown in Fig. 7. In second stage of solution tree both supplier and machine variation are checked by active concentration chart, for this batch of 100 parts fed to three machines. Input is eliminated because only variable available for input is supplier to supplier variation and it doesn't show large contrast from active concentration chart shown in Table 1. In process only variation showing large contrast from clue generation is machine to machine variation. Machine 3 was giving more rejections than other two machines, hence further focus is given to machine 3 .

Table 1. Active concentration chart

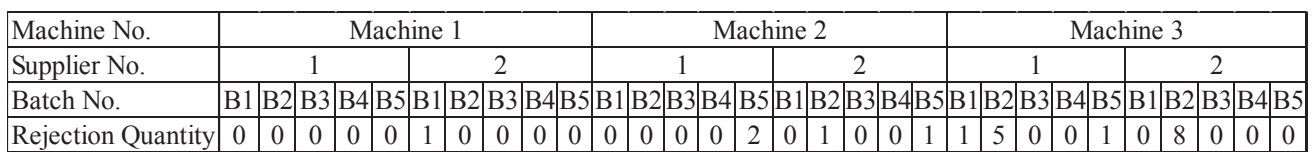

In machine 3, possible variations are time to time, tool to tool and holder (tool holder) to holder. Time to time variation is checked by collecting rejection data for one month as shown in Fig. 8, which doesn't give any clear contrast hence it is eliminated.

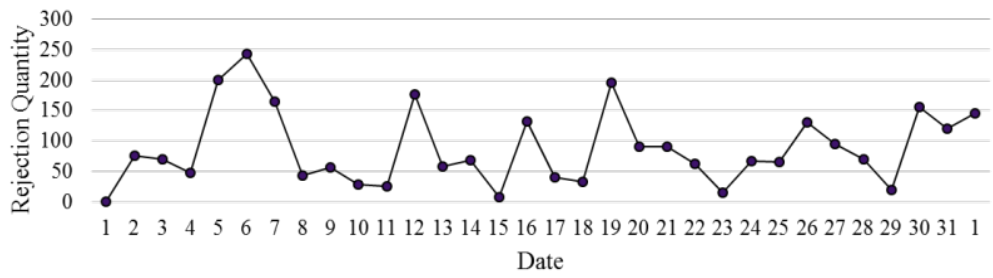

Fig. 8. Time to time rejection

For every 100 parts tool life is getting over and tool is changed automatically. Tool to tool variation is checked by collecting rejection data for multiple tools for its whole life shown in Table 2.

Table 2. Tool to Tool rejection

\begin{tabular}{|c|c|c|c|c|c|}
\hline \multicolumn{3}{|c|}{ Shift 1 } & \multicolumn{3}{c|}{ Shift 2 } \\
\hline Tool 1 & Tool 2 & Tool 3 & Tool 1 & Tool 2 & Tool 3 \\
\hline 2 & 1 & 2 & 1 & 1 & 2 \\
\hline
\end{tabular}


To check the tool holder variation, holders of best machine (Machine 1) and worst machine (Machine 3) are interchanged to check the effect (best and worst machines are selected on the basis of active concentration chart). From this exercise it is observed that rejection behaviour of machine 1 and machine 3 is reversed. This clearly indicates large contrast hence for further study both holders are disassembled. It is observed that holder associated with more rejections has an additional axial wear of $0.15 \mathrm{~mm}$ in tool holder assembly than new tool holder assembly. It is found that due to wear in tool holder assembly tool lift off value is ineffective, and hence tool holder wear is the root cause candidate.

\section{Results and Discussion}

B vs. W is technique in Shainin method to verify root cause. For confirmation of root cause candidate B vs. W test was conducted. For this test, three parts are fed to machine for conical grinding operation with worn out tool holder as batch 1 and three parts are fed to same machine with new tool holder as batch 2. Result of this exercise is shown in Fig.9. Where Green $\mathrm{Y}$ is surface roughness (profile peak), for which maximum acceptable value is $2 \mu \mathrm{m}$. It is observed that parts fed to the worn out tool holder gives all worst of worst parts and parts fed to new tool holder gives all best of best parts. B vs. W test indicates that Red X candidate drives Green Y hence axial wear in tool holder is confirmed as root cause for rough conical grinding problem.
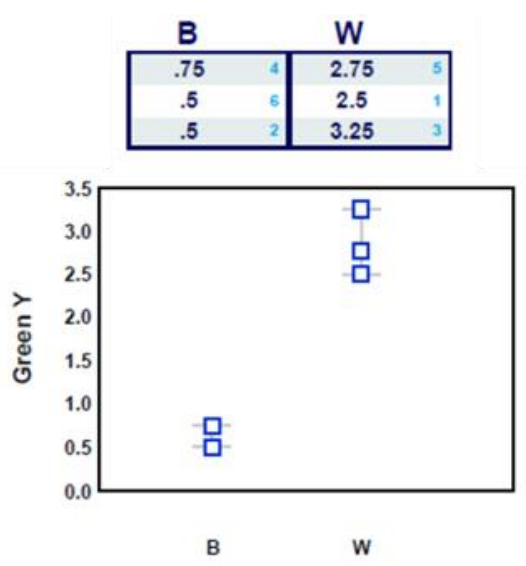

\begin{tabular}{|ll|}
\hline Required end count & 6 \\
Measured end count & 6 \\
$\begin{array}{l}\text { Is B better } \\
\text { than W? }\end{array}$ & yes \\
Test type & One tailed, smaller is better \\
Confidence & 0.95 \\
\hline
\end{tabular}

Fig. 9. B vs. W test

Contrast between good and bad parts speeds the Red X hunt which is important part of clue generation in Shainin method of problem solving. It is observed that Shainin ${ }^{\circledR}$ methodology can be successfully used in manufacturing related problems which helps to converge from large number of variables to most influential variable (root cause) by systematic use of Shainin tools.

\section{Conclusions}

Experimental investigation by Shainin method revealed additional axial wear of $0.15 \mathrm{~mm}$ in tool holder assembly is root cause (Red X) for HP port rough grinding issue of cylinder head. Wear in tool holder assembly makes tool lift off value ineffective, which restricts 
flow of oil between grinding tool and HP port surface during tool lift off. Identifying contrast between good and bad parts which is basic philosophy of Shainin method helps not just in clue generation but to identify the variables. Tools like active concentration chart and Isoplot in Shainin method helped eliminating variables and converging to most influential variable. B vs. W test helped to validate root cause candidate. Further in this study root cause identified can be eliminated by applying design of experiments by taking multiple levels of tool lift off as variable.

\section{References}

1. J. D. Mast, J. Lokkerbol, Int. J.Prod. Econ. 139, 604, (2012)

2. R. D. Shainin, Qty. Engg. 5, 433-448, (1993)

3. P Bhaskara, R Prasad, K M Subbaiah, G L Shekar, IJMET, 5, 200, (2014)

4. N. Logothetis, Qty \& Relia. Engg. Int.6, 195, (1990)

5. S. H. Steiner, R. J. Mackay, J. S. Ramberg, Qty. Engg. 20, 19, (2008)

6. A. J. Jegadeesan, L. Karunamoorthy, N. Arunkumar, JMST 29, 1731, (2015)

7. A Prashar, Prod. Plan. Ctrl. 27, 83, (2016) 